Pacific Journal of Mathematic 


\title{
SOME REMARKS ON CLIFFORD'S THEOREM AND THE SCHUR INDEX
}

\author{
G. J. JANUSZ
}

\begin{abstract}
Some time ago Clifford described the behavior of an irreducible representation of a finite group when it is restricted to a normal subgroup. One interesting case in this description requires that the representation be written in an algebraically closed field. In this note we shall consider this case when the field is "small". We describe conditions under which an irreducible representation decomposes as the tensor product of two projective representations. Our approach uses certain subalgebras of the group algebra and the course of the discussion makes it fairly easy to keep track of the division algebras that appear. Hence we obtain some information about the Schur index. We apply this information to the case where the group is a semi-direct product $P A$ of a $p$-group $P$ and a normal cyclic group $A$. If $\mathscr{F}$ is an algebraic number field and $\chi$ an absolutely irreducible character of $P A$, then there normal subgroups $P_{1} \supseteqq P_{2} \supseteqq P_{3}$ of $P$ which contain $C_{P}(A)$ such that the Schur index $m-(\chi)$ of $\chi$ over $\mathscr{F}$ divides $2\left[P_{1}: P_{2}\right] e$ where $e$ is the exponent of $P_{2} / P_{3}$. The factor 2 can be omitted if $p \neq 2$. Some conditions are available to restrict the $P_{i}$ further.
\end{abstract}

1. Preliminaries. In this section we summarize the results about the Schur index and Clifford's theory that will be used later.

Let $G$ be a finite group, $\mathscr{F}$ a field of characteristic zero, $M$ an irreducible $\mathscr{F}(G)$-module with character $\theta$.

(1.1) There are absolutely irreducible (complex-valued) characters $\chi_{1}, \cdots, \chi_{k}$ such that $\theta=m\left(\chi_{1}+\cdots+\chi_{k}\right)$.

(1.2) Let $\mathscr{F}\left(\chi_{i}\right)$ denote the field generated over $\mathscr{F}$ by the values of $\chi_{i}$ on $G$. Then $\mathscr{F}\left(\chi_{1}\right)$ is a normal extension of $\mathscr{F}$ and for each $i=1, \cdots, k$, there is a unique element of the Galois group of $\mathscr{F}\left(\chi_{1}\right)$ over $\mathscr{F}$ which carries $\chi_{1}$ to $\chi_{i}$. In particular $\left(\mathscr{F}\left(\chi_{1}\right): \mathscr{F}\right)=k$.

(1.3) The integer $m$ is called the Schur index of $\chi_{1}$ over $\mathscr{F}$ and is denoted by $m_{\mathscr{T}}\left(\chi_{1}\right)$. The division algebra $D=\operatorname{End}_{\mathscr{T}(G)}(M)$ has center isomorphic to $\mathscr{F}\left(\chi_{1}\right)$ and the dimension of $D$ over its center is $m^{2}$.

One remark on terminology. A matrix ring over $D$ is said to have index $m$ if $D$ has dimension $m^{2}$ over its center. 
The proofs of these statements are available in several places; see for example Curtis and Reiner [2] or Fein [5].

Now let $H$ be a normal subgroup of $G$. Clifford's theory tells how $M$ behaves as a module over $\mathscr{F}(H)$.

(1.4) $\quad M_{H} \cong n\left(V_{1} \oplus \cdots \oplus V_{s}\right)$ where the $V_{i}$ are mutually nonisomorphic irreducible $\mathscr{F}(H)$-modules, conjugate under the action of $G$. Here the coefficient $n$ means direct sum of $n$ copies of $V_{1} \oplus \cdots \oplus V_{s}$.

(1.5) Let $I_{1}=\left\{x \in G \mid x V_{1} \cong V_{1}\right.$ as $\mathscr{F}(H)$-modules $\}$. Then there exists an irredubible $\mathscr{F}\left(I_{1}\right)$-module $W_{1}$ such that $\left(W_{1}\right)_{H} \cong n V_{1}$ and the induced module $W_{1}^{G} \cong M$.

In the case where $s>1, I_{1}$ is a proper subgroup of $G$ and the original module is induced from the $\mathscr{F}\left(I_{1}\right)$-module. Hence some questions can be answered by induction. In case $s=1$ there is no induction but in its place we have the following.

(1.6) Suppose $\mathscr{F}$ is algebraically closed and $s=1$ in (1.4). Then the representation afforded by $M$ decomposes into the tensor product of two (irreducible) projective representations of $G$ one of which can be viewed as a projective representation of $G / H$. The one representation has dimension the same as the dimension of $V_{1}$, the other, has dimension $n$.

2. Clifford's Theorem in the general case. We shall continue to use the notation introduced in $\S 1$. However we assume $M_{I I} \cong n V$ with $V=V_{1}$ in (1.4). We shall made one assumption that will simplify the following discussion considerably. Namely we assume that $\mathscr{F}$ contains the values of the character $\chi_{1}$. Then in view of (1.2) we have $\theta=m \chi$ where $\chi=\chi_{1}$ in (1.1).

Let $V$ have character $\gamma$ and suppose

$$
\gamma=m_{H}\left(\varphi_{1}+\cdots+\varphi_{t}\right)
$$

is the decomposition of $\gamma$ into absolutely irreducible characters of $H$.

In the group algebra $\mathscr{F}(G)$ let $e(\theta)$ denote the central idempotent which acts as the identity on $M$ and such that $\mathscr{F}(G) e(\theta)$ is a simple algebra; let $e(\gamma)$ denote the centrally primitive idempotent in $\mathscr{F}(H)$ corresponding to $V$. The condition that $M_{H} \cong n V$ implies $\gamma(h)=$ $\gamma\left(x^{-1} h x\right)$ for all $h$ in $H$ and $x$ in $G$. It follows that $e(\gamma)$ belongs to the center of $\mathscr{F}(G)$. Also $e(\theta) e(\gamma) \neq 0$ because both act as the identity on $V$ so $e(\theta) e(\gamma)$ is a nonzero central idempotent in $\mathscr{F}(G) e(\theta)$. By simplicity we must have $e(\theta) e(\gamma)=e(\theta)$. Thus multiplication by $e(\theta)$ sends the simple algebra $\mathscr{F}(H) e(\gamma)$ onto the nonzero subalgebra 
$\mathscr{F}(H) e(\theta)$ of $\mathscr{F}(G) e(\theta)$ both having the same identity, $e(\theta)$. We note that by (1.3) and (2.1) the center $\mathscr{L}$ of $\mathscr{F}(H) e(\theta)$ is isomorphic to $\mathscr{F}\left(\varphi_{1}\right)$. So we have proved

LemMA 2.2. $\mathscr{F}(H) e(\theta)$ is a simple algebra with center $\mathscr{L}$ isomorphic to $\mathscr{F}\left(\varphi_{1}\right)$.

Each element of $G$ acts by conjugation on $\mathscr{F}(H) e(\theta)$ since $e(\theta)$ is central and $H$ is a normal subgroup. Thus $G$ also acts on the center $\mathscr{L}$ of $\mathscr{F}(H) e(\theta)$ as a group fixing $\mathscr{F}$. Let $I$ denote the kernel of the action of $G$ on $\mathscr{L}$ so that $I$ is normal in $G$ and $G / I$ is a group of $\mathscr{F}$-automorphisms of $\mathscr{L}$. Let $\left\{y_{i}\right\} 1 \leqq i \leqq r$ be a set of representatives of the cosets of $I$ in $G$ and let $y_{i}$ induce the automorphism $\sigma_{i}$ oh $\mathscr{L}$.

Lemma 2.3. The elements $y_{i} e(\theta)$ are independent over $\mathscr{F}(I) e(\theta)$ and $\mathscr{F}(G) e(\theta)=\sum \mathscr{F}(I) e(\theta) y_{i}$.

Proof. Suppose there exist elements $\left\{\alpha_{j}\right\}$ in $\mathscr{F}(G) e(\theta)$ which centralize $\mathscr{L}$ and with the properties

(a) $\sum_{j=1}^{s} \alpha_{j} y_{j} e(\theta)=0$.

(b) $\alpha_{j} \neq 0$ for each $j$.

(c) The integer $s$ is minimal with respect to (a) and (b). Then for any $z$ in $\mathscr{L}$ we have $\sum \alpha_{j} z y_{j}=\sum \alpha_{j} y_{j} z=0$. Then $y_{j}$ induces $\sigma_{j} \mathscr{L}$ so

$$
\sum_{j=1}^{s} \alpha_{j}\left(z-\sigma_{j}(z)\right) y_{j}=0 .
$$

There is no loss of generality in assuming that $y_{1}=1$ since a change of coset representatives can always bring this about. Thus $z-\sigma_{1}(z)=$ 0 and the relation (2.4) has fewer than $s$ nonzero terms. By the choice of $s$ it follows $\alpha_{j}\left(z-\sigma_{j}(z)\right)=0$ for each $j$ and all $z$ in $\mathscr{L}$. If $j>1$ then $\sigma_{j} \neq$ identity so there exists $z$ in $\mathscr{L}$ with $\sigma_{j}(z) \neq z$. But then $\alpha_{j}=0$ contrary to (b). Hence $s=1$ but this is also contrary to (b). Thus no such relation exists. Since $\mathscr{F}(I) e(\theta)$ centralizes $\mathscr{L}$ we have proved the independence of the $y_{i} e(\theta)$ over $\mathscr{F}(I) e(\theta)$. The second part of the lemma is clear.

COROLlary 2.5. $\mathscr{F}(I) e(\theta)$ is the full centralizer of $\mathscr{L}$ in $\mathscr{F}(G) e(\theta)$ so $\mathscr{F}(I) e(\theta)$ is a simple algebra with center $\mathscr{L}$ and $d i$ mension $(\chi(1) / t)^{2}$ over $\mathscr{L}$.

Proof. The lemma shows the $y_{i} e(\theta)$ are independent over the 
centralizer of $\mathscr{L}$ and so the proper inclusion of $\mathscr{F}(I) e(\theta)$ into the centralizer of $\mathscr{L}$ would make the equality $\mathscr{F}(G) e(\theta)=\sum \mathscr{F}(I) e(\theta) y_{i}$ impossible. The remaining statements follow from Albert, Theorem 12, page 53 [1] and the facts that $(\mathscr{F}(G) e(\theta): \mathscr{F})=\chi(1)^{2}$ and $(\mathscr{L}: \mathscr{F})=t(\operatorname{see}(1.2)$ and $(2.2))$.

Corollary 2.6. $[G: I]=t$ so $G / I \cong$ Galois group (L/L/F).

Proof. The result follows at once if we use (2.3) and (2.5) to compute the dimension of $\mathscr{F}(G) e(\theta)$ over $\mathscr{F}$ along with the fact that this dimension is $\chi(1)^{2}$.

Now let $\chi \mid H=a\left(\varphi_{1}+\cdots+\varphi_{t}\right)$. Since $\theta=m \chi$ and $\theta \mid H=n \gamma$, equation (2.1) implies $a=n m_{H} / m$. Let $I_{1}$ denote the inertial group of $\varphi_{1}$; that is

$$
I_{1}=\left\{x \in G \mid \varphi_{1}(h)=\varphi_{1}\left(x^{-1} h x\right) \text { for all } h \in H\right\} .
$$

CoRollary 2.7. $I_{1}=I$.

Proof. The irreducible characters of $H$ appearing in $\chi \mid H$ are conjugate under the action of $G$ so that $\left[G: I_{1}\right]=t=[G: I]$. Thus it is sufficient to show $I_{1} \subseteq I$. Let $\mathscr{F}_{1}=\mathscr{F}\left(\varphi_{1}\right)$ and notice that in $\mathscr{F}_{1}(H)$ we have $e(\gamma)=e\left(\varphi_{1}\right)+\cdots+e\left(\varphi_{t}\right)$ where $e\left(\varphi_{1}\right)$ is the central idempotent of $\mathscr{F}_{1}(H)$ corresponding to $\varphi_{i}$. Recall from above that $e(\gamma) e(\theta)=e(\theta)$ so

$$
\mathscr{F}(H) e(\theta)=\mathscr{F}(H)\left(e\left(\varphi_{1}\right)+\cdots+e\left(\varphi_{t}\right)\right) e(\theta) \stackrel{R}{\longrightarrow} \mathscr{F}_{1}(H) e\left(\varphi_{1}\right) e(\theta)
$$

where $R$ denotes right multiplication by $e\left(\varphi_{1}\right)$. The map $R$ is a ring isomorphism. One point requires further comment. The obvious range of $R$ is $\mathscr{F}(H) e\left(\varphi_{1}\right) e(\theta)$ rather than $\mathscr{F}_{1}(H) e\left(\varphi_{1}\right) e(\theta)$. However we can prove these are equal in the following way. Certainly $\mathscr{F} \cong \mathscr{F}_{1}$ so $\mathscr{F}(H) e\left(\varphi_{1}\right) e(\theta) \subseteq \mathscr{F}_{1}(H) e\left(\varphi_{1}\right) e(\theta)$. We prove equality by computing the $\mathscr{F}$-dimension of both sides. Since multiplication by $e(\theta)$ gives an $\mathscr{F}_{1}$-algebra isomorphism of $\mathscr{F}_{1}(H) e\left(\varphi_{1}\right)$ onto $\mathscr{F}_{1}(H) e\left(\varphi_{1}\right) e(\theta)$ we see the latter algebera has $\mathscr{F}_{1}$-dimension equal to $\varphi_{1}(1)^{2}$ and $\mathscr{F}$-dimension $t \varphi_{1}(1)^{2}$. To compute $\mathscr{F}$-dimension of $\mathscr{F}(H) e\left(\varphi_{1}\right) e(\theta)$ we first note $\mathscr{F}$ dimension of $\mathscr{F}(H) e(\gamma) e(\theta)$ equals $\mathscr{F}_{1}$-dimension of $\mathscr{F}_{1}(H) e(\gamma) e(\theta)$ because the latter algebra is obtained by extending the scalar field from $\mathscr{F}$ to $\mathscr{F}_{1}$. Now the algebra $\mathscr{F}_{1}(H) e(\gamma) e(\theta)$ equals $\sum \mathscr{F}_{1}(H) e\left(\varphi_{i}\right) e(\theta)$ and this has $\mathscr{F}$-dimension $t \varphi_{1}(1)^{2}$ as we wanted.

For an element $x$ in $I_{1}$ we have $x^{-1} e\left(\varphi_{1}\right) x=e\left(\varphi_{1}\right)$ so the map $R$ commutes with the action of $I_{1}$ on the two algebras in question. It is clear that $\mathscr{F}_{1}$ is the center of $\mathscr{F}_{1}(H) e\left(\varphi_{1}\right) e(\theta)$ because $\mathscr{F}_{1}(H) e\left(\varphi_{1}\right)$ is simple with center $\mathscr{F}_{1}$. Moreover $I_{1}$ fixes $\mathscr{F}_{1}$ since $\mathscr{F}_{1}$ is the scalar 
field. But $R$ maps $\mathscr{L}$ onto $\mathscr{F}_{1}$ since the center is preserved. Thus $I_{1}$ fixes $\mathscr{L}$ and $I_{1} \subseteq I$.

We can now give the analogue of (1.6).

THEOREM 2.8. The representation of $I$ into $\mathscr{F}(I) e(\theta)$ given by $x \rightarrow x e(\theta)$ decomposes into the tensor product of two projective representations over $\mathscr{L}, U_{x}$ and $T_{x}$, which map $I$ onto $\mathscr{F}(H) e(\theta)$ and $\mathscr{C}$ respectively where $\mathscr{C}$ is the centralizer of $\mathscr{F}(H) e(\theta)$ in $\mathscr{F}(I) e(\theta)$. The dimensions over $\mathscr{L}$ of these two algebras are $\varphi_{1}(1)^{2}$ and $a^{2}$ respectively.

Proof. Each element of $I$ acts by conjugation on $\mathscr{F}(H) e(\theta)$ in such a way that the center is left fixed. Every such automorphism of this simple algebra is inner. Hence for each $x$ in $I$ there is an element $U_{x}$ in $\mathscr{F}(H) e(\theta)$ such that $x^{-1} w x=U_{x}^{-1} w U_{x}$ for every $w$ in $\mathscr{F}(H) e(\theta)$. Clearly the element $\alpha(x, y)=U_{x} U_{y} U_{x y}^{-1}$ induces the identity automorphism so $\alpha(x, y)$ is in $\mathscr{L}$ and it follows that $x \rightarrow U_{x}$ is a projective representation of $I$ with factor set $\alpha$ having values in $\mathscr{L}$.

Now let $\mathscr{C}$ denote the centralizer of $\mathscr{F}(H) e(\theta)$ in $\mathscr{F}(I) e(\chi)$. By Theorem 13, page 53 of [1] it follows

$$
\mathscr{F}(I) e(\theta)=\mathscr{F}(H) e(\theta) \cdot \mathscr{C} \cong \mathscr{F}(H) e(\theta) \bigotimes \mathscr{C}
$$

because $\mathscr{F}(H) e(\theta)$ and $\mathscr{F}(I) e(\theta)$ both have center $\mathscr{L}$. We also know then that $\mathscr{C}$ is simple with center $\mathscr{L}$. Set $T_{x}=x U_{x}^{-1}$ so $T_{x}$ is in $\mathscr{C}$. Then $x \rightarrow T_{x}$ is a projective representation of $I$ with factor set $\alpha^{-1}$ and $x e(\theta)=U_{x} \otimes T_{x}$ as required.

We know $(\mathscr{F}(H) e(\theta): \mathscr{L})=\varphi_{1}(1)^{2}$ and from (2.5) that

$$
(\mathscr{F}(I) e(\theta): \mathscr{L})=(\chi(1) / t)^{2} .
$$

We also have $\chi(1)=a t \varphi_{1}(1)$ so that we easily obtain from (2.9) the dimension of $\mathscr{C}$ over $\mathscr{C}$ is $a^{2}$.

Corollary 2.10. If $\mathscr{F}\left(\varphi_{1}\right)=\mathscr{F}$ then the algebra $\mathscr{F}(G) e(\theta)$ decomposes as the tensor product $\mathscr{F}(H) e(\theta) \otimes \sigma$ and the representation offered by $M$ decomposes as the tensor product of two projective representations into $\mathscr{F}(H) e(\theta)$ and $\mathscr{C}$ respectively.

Proof. Since $\mathscr{F}\left(\varphi_{1}\right)=\mathscr{F}$ we have $\mathscr{C}=\mathscr{F}$ and so $G=I$ by (2.6).

3. The Abelian case. We continue with the same situation except that we now suppose $G / H$ is abelian.

Since $I$ is the subgroup fixing $\varphi_{1}$, there is an absolutely irreducible 
character $\zeta$ of $I$ such that $\zeta^{G}=\chi$ and $\zeta \mid H=a \varphi_{1}$. Now $I \triangleleft G$ so that in fact we obtain

$$
\chi\left|I=\zeta_{1}+\cdots+\zeta_{t}, \zeta_{i}\right| H=a \varphi_{i}
$$

where the $\zeta_{i}$ are irreducible characters of $I$ conjugate under the action of $G$ and $\zeta=\zeta_{1}$.

LemmA 3.2. There exists a subgroup $J$ of $I$ containing $H$ and an irreducible character $\tau$ of $J$ such that $\zeta \mid J=a \tau$ and $\tau^{I}=a \zeta$. Then also $\tau \mid H=\varphi_{1}$ and $[I: J]=a^{2}$.

Proof. Let $\Lambda$ be the set of linear characters, $\lambda$, of $I / H$ such that $\zeta \lambda=\zeta$ and set $J=\cap \operatorname{ker} \lambda$ as $\lambda$ runs through $\Lambda$. We show this $J$ has the required properties.

Let $\rho$ denote the character of the regular representation of $I / H$. It is a straight-forward computation to verify that $a \varphi_{1}^{I}=\zeta \rho$ since both sides are 0 off $H$ and equal to $a[I: H] \varphi_{1}$ on $H$. By Frobenius reciprocity $\zeta$ has multiplicity $a$ in $\phi_{1}^{I}$ and so has multiplicity $a^{2}$ in $\zeta \rho$. But $\rho$ is the sum of the distinct linear characters of $I / H$ and so there are exactly $a^{2}$ linear characters $\lambda$ such that $\zeta \lambda=\zeta$. Hence $|\Lambda|=a^{2}$. Note that $\Lambda$ is also a subgroup of the group of linear characters of $I / H$ so by the duality theory of abelian groups we obtain $[I: J]=a^{2}=|\Lambda|$.

Now let $\tau$ be an irreducible character of $J$ contained in $\zeta \mid J$ with multiplicity $b$ say. If $[I: J]$ is a prime then either $\tau^{I}=\zeta$ or $\tau^{I}=$ $\zeta \Psi_{1}+\zeta \Psi_{2}+\cdots$ where the $\Psi_{i}$ are the linear characters of $I / J$. Because $I / J$ is abelian we use induction to find in the general case that $\tau^{I}$ is a sum of characters $\zeta \Psi$ where $\Psi$ is a linear character of $I / J$. But every such linear character is in $\Lambda$ so it follows $\tau^{I}=b \zeta$. Also there is an integer $c$ such that $\tau \mid H=c \varphi_{1}$ because $\tau \mid H$ is contained in $\zeta \mid H=$ $a \varphi_{1}$. Now compute degrees of the characters involved.

$$
\tau^{I}(1)=|I: J| \tau(1)=a^{2} c \varphi_{1}(1)=b \zeta(1)=b a \varphi_{1}(1) .
$$

So we obtain $a c=b$.

The decomposition of $\zeta$ on $J$ has the form

$$
\zeta \mid J=b\left(\tau+\tau_{2}+\cdots+\tau_{k}\right)
$$

so we find

$$
\zeta(1)=a \varphi_{1}(1)=b k \tau(1)=b k c \varphi_{1}(1) .
$$

Thus $a=b k c$ and along with $a c=b$ we find $k=c=1$ and $a=b$ which proves the lemma. 
Notice that $\tau \mid H=\varphi_{1}$ implies that $\tau$ has precisely $t$ conjugates under $G, \tau=\tau_{1}, \cdots, \tau_{t}$ and the numbering can be arranged to satisfy $\tau_{i} \mid H=\varphi_{i}$. Then also $\chi \mid J=a\left(\tau_{1}+\cdots+\tau_{t}\right)$. We shall make use of this in the next result.

Lemma 3.3. $\mathscr{F}(J) e(\theta)$ is a simple algebra with center $\mathscr{L}$.

Proof. The ring $\mathscr{F}(J) e(\theta)$ is semi-simple so simplicity will follow if we show it has only one irreducible module (up to isomorphism). Any irreducible $\mathscr{F}(J) e(\theta)$ module, $W$, is isomorphic to a direct summand of $M_{J}$ because $M$ is the unique isomorphism type of $\mathscr{F}(G) e(\theta)$ module. Let $\mu$ be the character of $W$. The character $\gamma$ must appear in $\mu \mid H$ since $\gamma$ is the only character of an irreducible $\mathscr{F}(H) \mathrm{e}(\theta)$ module in $M_{H}$. Thus $\mu \mid H$ contains each $\varphi_{i}$. Moreover the absolutely irreducible characters in $\mu$ must appear in $\chi \mid J$. By the remark above the lemma, every $\tau_{i}$ appears in $\mu$. Thus $\mu$ is invariant under $G$ and it follows $M_{J}=k \cdot W$ for some $k$. Hence $\mathscr{F}(J) e(\theta)$ has only one irreducible module. We find also that $\mathscr{F}(J) e(\theta)$ is isomorphic to $\mathscr{F}(J) e(\mu)$ and its center is isomorphic to $\mathscr{F}(\tau)$. The equations $\zeta \mid J=$ $a \tau$ and $\tau^{I}=a \zeta$ imply $\mathscr{F}(\tau)=\mathscr{F}(\zeta)$. But then $\mathscr{F}(\zeta)$ is isomorphic to the center of $\mathscr{F}(I) e(\theta)$ so by $(2.5)$ and $(2.2) \mathscr{F}(\zeta) \cong \mathscr{F}\left(\varphi_{1}\right)$. These are isomorphisms over $\mathscr{F}$ so in fact $\mathscr{F}(\zeta)=\mathscr{F}\left(\varphi_{1}\right)$ because both are normal extensions. Hence the center of $\mathscr{F}(J) e(\theta)$ is isomorphic to $\mathscr{L}$. Because of the inclusions

$$
\mathscr{L} \subseteq \mathscr{F}(H) e(\theta) \leqq \mathscr{F}(J) e(\theta)
$$

and the fact that $\mathscr{L}$ centralizes $\mathscr{F}(J) e(\theta)$ we have $\mathscr{L}=$ center $\mathscr{F}(J) e(\theta)$.

Now that we know $\mathscr{F}(I) e(\theta)$ and $\mathscr{F}(J) e(\theta)$ have the same centers we obtain a decomposition

$$
\mathscr{F}(I) e(\theta)=\mathscr{F}(J) e(\theta) \cdot \mathscr{C}_{J} \cong \mathscr{F}(J) e(\theta) \otimes \mathscr{C}_{J}
$$

where $\mathscr{C}_{J}$ is the centralizer of $\mathscr{F}(J) e(\theta)$. If we apply (2.8) with $J$ in place of $H$ we find dimension $\mathscr{C}_{J}$ over $\mathscr{L}$ is $a^{2}$. This is the same $a$ that appears for $H$ because $\chi \mid J=a\left(\tau_{1}+\cdots+\tau_{t}\right)$. It is clear that $\mathscr{C}$ (the centralizer of $\mathscr{F}(H) e(\theta)$ ) contains $\mathscr{C}_{J}$ and so by dimension count we find $\mathscr{C}_{J}=\mathscr{C}$. From this it follows that $\mathscr{F}(J) e(\theta)=$ $\mathscr{F}(H) e(\theta)$. This makes it possible to adjust the projective representations $U$ and $T$ so that $U_{x}=x e(\theta)$ if $x$ is in $J$ and $T$ is constant on the cosets of $J$.

We are now able to identify the algebra $\mathscr{C}$.

Proposition 3.5. Let $\mathscr{F}_{1}$ denote $\mathscr{F}\left(\varphi_{1}\right)$. The algebra $\mathscr{C}$ is iso- 
morphic to a twisted group alegebra $\mathscr{F}_{1}(I / J)_{\beta}$ for some factor set $\beta$ on $I / J$ with values in $\mathscr{F}_{1}$.

Proof. Recall that the twisted group algebra $\mathscr{F}_{1}(I / J)_{\beta}$ has a basis $\left\{t_{x} \mid x \in I / J\right\}$ such that $t_{x} t_{y}=\beta(x, y) t_{x y}$. The modification of $U$ above allows us to view $T$ as a projective representation of $I / J$. Then the correspondence $t_{x} \rightarrow T_{x}$ for $x$ in $I / J$ induces a homomorphism from $\mathscr{F}_{1}(I / J)_{\beta}$ into $\mathscr{C}$ provided we have fixed identification of $\mathscr{F}_{1}$ with $\mathscr{L}$ and $\beta=\alpha^{-1}$ on $I / J$. If we show this homomorphism is onto $\mathscr{C}$, we will be finished because both algebras have dimension $a^{2}$ over $\mathscr{F}_{1}$. From equation (3.4) it follows

$$
\mathscr{F}(I) e(\theta)=\sum_{x \in I / J} \mathscr{F}(J) e(\theta) \otimes T_{x}
$$

becase the right side contains $\mathscr{L}$ along with every $y e(\theta)$ for $y$ in $I$. It follows that the $T_{x}$ span $\mathscr{C}$ over $\mathscr{L}$ (because the tensor product is taken over $\mathscr{L}$ ) and hence the homomorphism above is onto $\mathscr{C}$.

Let $A$ denote $I / J$. The fact that $\mathscr{F}_{1}(A)_{\beta}$ is simple with center $\mathscr{F}_{1}$ imposes restrictions on $A$ and one can say quite a lot about $\mathscr{F}_{1}(A)_{\beta}$. We shall give a brief sketch of the results of DeMeyer [3] which are relevant.

Consider the function $\eta(a, b)=\beta(a, b) / \beta(b, a)$. Because $A$ is abelian and $\beta$ is a factor set, it follows that $\eta$ is a (multiplicative) skew bilinear form from $A \times A$ to the multiplicative group of $\mathscr{F}_{1}$. This is $\eta(a b, c)=\eta(a, c) \eta(b, c)$ and $\eta(a, b)=\eta(b, a)^{-1}$. Because $\mathscr{F}_{1}$ is the center of $\mathscr{F}_{1}(A)_{\beta}, \eta$ is nondegenerate; that is $\eta(a, A)=1$ holds only for $a=$ 1. In a way similar to the method of decomposing a vector space admitting a skew bilinear form, one decomposes $A$ into the direct sum of "hyperbolic planes". The result is the following.

THEOREM (DeMeyer [3]). Let $\mathscr{F}_{1}(A)_{\beta}$ be central simple over $\mathscr{F}_{1}$. Then $A$ decomposes as

$$
A=\left(C_{11} \times C_{12}\right) \times\left(C_{21} \times C_{22}\right) \times \cdots \times\left(C_{r 1} \times C_{r 2}\right)
$$

where $C_{i j}$ is cyclic of prime-power order and $C_{i 1} \cong C_{i 2}$. The function $\eta$ remains nondegenerate on $C_{i 1} \times C_{i 2}$ and the subalgebras $\mathfrak{A}_{i}=$ $\mathscr{F}_{1}\left(C_{i 1} \times C_{i 2}\right)_{\beta}$ are central simple over $\mathscr{F}_{1}$. Finally we have the decomposition

$$
\mathscr{F}_{1}(A)_{\beta} \cong \mathfrak{N}_{1} \otimes \cdots \otimes \mathfrak{N}_{r}
$$

This decomposition allows us to get information about the index of the algebra $\mathscr{F}_{1}(A)_{\beta}$. We must now restrict $\mathscr{F}$ to be an algebraic number field. Now the Brauer-Hasse-Noether theorem can be applied. 
It tells us the index of a finite dimensional division algebra over $\mathscr{F}$ is equal to its exponent; that is the order of its class in the Brauer group of the center of the division algebra.

The index of each $\mathfrak{A}_{i}$ is a divisor of $\left|C_{i 1}\right|$ since dimension of $\mathfrak{A}_{i}$ over $\mathscr{F}_{1}$ is $\left|C_{i 1}\right|^{2}$. Thus the index of $\mathscr{F}_{1}(A)_{\beta}$ divides the least common multiple of the indices of the $\mathfrak{A}_{i}$ since the exponent of $\mathfrak{A}_{1} \otimes \cdots \otimes \mathfrak{A}_{r}$ divides the 1.c.m. of the exponents of the $\mathfrak{A}_{i}$. This in turn divides the 1.c.m. of the numbers $\left|C_{i 1}\right| 1 \leqq i \leqq r$ and this number is precisely the exponent of $A$. So we have the

Proposition 3.6. The index of the algebra $\mathscr{C}$ divides the exponent of $I / J$.

Theorem 3.7. The Schur index $m_{\mathscr{T}}(\chi)$ of $\chi$ divides

$$
[G: I] \cdot 1 . c . m .\left\{m_{\mathscr{T}}\left(\varphi_{1}\right), \text { exponent }(I / J)\right\} \text {. }
$$

Proof. We have $\mathscr{F}_{1} \otimes \mathscr{F}(G) e(\theta)$ equivalent to $\mathscr{F}(I) e(\theta)$ in the Brauer group of $\mathscr{F}_{1}$ because by Theorem 16, page 56 of [1],

$$
\mathscr{F}_{1} \otimes \mathscr{F}(G) e(\theta) \cong \mathscr{C}_{t}(\mathscr{F}) \bigotimes_{\mathscr{F}} \mathscr{F}(I) e(\theta) \text {. }
$$

Hence by Theorem 20, page 59 of [1] the factor by which the index has been reduced after extending the field to $\mathscr{F}_{1}$ must divide $\left[\mathscr{F}_{1}: \mathscr{F}\right]$. By (2.6) this number is $[G: I]$ so the index of $\mathscr{F}(G) e(\theta)$ divides $[G: I]$ times the index of $\mathscr{F}(I) e(\theta)$. By the decomposition of (2.9) we see the index of $\mathscr{F}(I) e(\theta)$ divides the least common multiple of the index of $\mathscr{F}(H) e(\theta)$ and the index of $\mathscr{C}$. The index of $\mathscr{F}(H) e(\theta)$ is $m_{\pi}\left(\varphi_{1}\right)$ so the result follows from (3.6).

There is a theorem of Brauer [2, Theorem 70.28] which shows that certain questions about the Schur index of an irreducible character for a finite group can be reduced to questions about $\mathscr{F}$-elementary groups. Recall that among other things an $\mathscr{F}$-elementary group is a semi-direct product $P A$ of a $p$-group $P$ and a normal cyclic $p^{\prime}$-group $A$. We now consider the case where $G=P A$ is such a semi-direct product (not necessarily $\mathscr{F}$-elementary however). Let $H=C_{G}(A)$ so that $H$ is normal and $G / H$ is abelian (because $A$ has an abelian automorphism group). Let $\chi$ be an absolutely irreducible character of $G$. In this situation we have the following.

Theorem 3.8. Assume $\mathscr{F}$ is an algebraic number field. Then there exists a chain of normal subgroups

$$
G \supseteqq G_{1} \supseteqq I \supseteqq J \supseteqq H
$$


such that the Schur index of $\chi$ over $\mathscr{F}$ divides $2\left[G_{1}: I\right] \cdot$ exponent $(I / J)$. The factor 2 can be omitted if $p \neq 2$ or if $p=2$ but $I \neq J$.

Proof. Since the Schur index will not change, assume $\mathscr{F}=$ $\mathscr{F}(\chi)$. Let $M_{0}$ be an irreducible $\mathscr{F}(G)$-module with character $\theta_{0}=$ $m \chi$. Suppose $M_{0} \mid H=f\left(V_{1} \oplus \cdots \oplus V_{r}\right)$ with the $V_{i}$ distinct irreducible $\mathscr{F}(H)$-modules. Let $G_{1}=\left\{x \in G \mid x V_{1} \cong V_{1}\right.$ as $\mathscr{F}(H)$-modules $\}$. Then there is an irreducible $\mathscr{F}\left(G_{1}\right)$-module $W_{1}$ such that $W_{1}^{a} \cong M_{0}$ and $W_{1} \mid H \cong f \cdot V_{1}$. Since $G / H$ is abelian we know $G_{1} \triangleleft G$ so $M_{0} \mid G_{1}=$ $W_{1} \oplus \cdots \oplus W_{r}$ where the $W_{i}$ are mutually nonisomorphic irreducible $\mathscr{F}\left(G_{1}\right)$-modules. Now for any nonzero $\delta$ in End ${ }_{\mathscr{S}(G)}\left(M_{0}\right)$ we have $\delta\left(W_{1}\right) \cong W_{1}$ and since the $W_{i}$ are nonisomorphic, $\delta\left(W_{1}\right)=W_{1}$. Hence we imbed End $\sigma_{(G)}\left(M_{0}\right)$ into End ${\widetilde{T}\left(G_{1}\right)}_{(}\left(W_{1}\right)$. Conversely the equation $W_{1}^{a} \cong M_{0}$ provides a natural imbedding of End ${ }_{\mathscr{S}\left(G_{1}\right)}\left(W_{1}\right)$ into $\operatorname{End}_{\mathscr{S}^{\prime}(G)}\left(M_{0}\right)$. Hence these two division algebras are isomorphic. Let $\chi \mid G_{1}$ contain the character $\zeta$ which also appears in the decomposition of the character for $W_{1}$. We have $m(\chi)=m_{\mathscr{J}}(\zeta)$ since these numbers represent the indices of the respective endomorphism rings above. Moreover $\mathscr{F}(\zeta)$ is the center of End ${ }_{\left.-\backslash G_{1}\right)}\left(W_{1}\right)$ so $\mathscr{F}=\mathscr{F}(\zeta)$. We may now apply (3.7) to $\mathscr{F}, G_{1}$, and $W_{1}$ in place of $\mathscr{F}, G, M$ and obtain

$$
m \cdot(\chi) \mid\left[G_{1}: I\right] 1 . c . m .\left\{\operatorname{exponent}(I / J), m_{\mathscr{T}}\left(\varphi_{1}\right)\right\}
$$

where again $\varphi_{1}$ is an irreducible character of $H$ contained in $\chi \mid H$. But $H=C_{P}(A) \times A$ is a nilpotent group so by Roquette's theorem [6], $m,\left(\varphi_{1}\right)=1$ or possibly 2 in case $p=2$. Even when $m_{\sigma}\left(\varphi_{1}\right)=2$ the 1.c.m. of exponent $(I / J)$ and $m,-\left(\varphi_{1}\right)$ will be exponent $(I / J)$ provided $I \neq J$. So the result follows.

CoROLlaRY 3.9. If $\chi \mid H=a\left(\varphi_{1}+\cdots+\varphi_{t}\right)$ then $m_{-}(\chi) \mid$ 2at where the 2 can be omitted if $p \neq 2$.

Proof. If $\chi \mid H=a\left(\varphi_{1}+\cdots+\varphi_{t}\right)$ then $\zeta \mid H=a\left(\varphi_{1}+\cdots+\varphi_{r}\right)$ where $r \mid t$. Thus $|I: J|=a^{2}$ and exponent $(I / J)$ divides $a$. The definition of $I$ yields $\left[G_{1}: I\right]=r$ so $\left[G_{1}: I\right] \mid t$. The result now follows from the theorem.

REMARKs. (a) It can happen that $m_{-}(\chi)=a t$. This is the case when $\chi$ is an irreducible character of degree 3 for the metacyclic group $\langle x, y\rangle$ where $x^{7}=y^{9}=1$ and $y^{-1} x y=x^{4}$.

(b) The application of DeMeyer's theorem shows the interest in twisted group algebras, $\mathscr{F}(G)_{\alpha}$, which are simple with center $\mathscr{F}$. A discussion of groups $G$ which admit such a factor set $\alpha$ can be found in [4]. 


\section{BIBLIOGRAPHY}

1. A. A. Albert, Structure of algebras, Amer. Math. Soc. Colloquium Publications, 24 (1939).

2. C. W. Curtis and I. Reiner, Representation theory of finite groups and associative algebras, Interscience, New York, 1962.

3. F. DeMeyer, Galois theory in separable algebras over commutative rings, Illinois J. Math. 10 (1966), 287-295.

4. F. DeMeyer and G.J. Janusz, Finite groups with an irreducible representation of large degree, Math. Z. 108 (1969), 145-153.

5. B. Fein, Representations of direct products of finite groups, Pacific J. Math. 20 (1967), 45-58.

6. P. Roquette, Realisierung von Darstellungen endlicher nilpotenter Gruppen, Archiv. der Math. 9 (1958), 241-250.

Received September 9, 1968.

UNIVERSITY OF ILLINOIS

URBANA, ILLINOIS 



\section{PACIFIC JOURNAL OF MATHEMATICS}

\section{EDITORS}

\author{
H. SAMELSON \\ Stanford University \\ Stanford, California 94305 \\ Richard Pierce \\ University of Washington \\ Seattle, Washington 98105
}

J. DUGUNDJI

Department of Mathematics

University of Southern California

Los Angeles, California 90007

BASIL GORDON*

University of California

Los Angeles, California 90024

\section{ASSOCIATE EDITORS}
E. F. BECKENBACH
B. H. NeUmanN
F. WOLF
K. YoSHIDA

\section{SUPPORTING INSTITUTIONS}

UNIVERSITY OF BRITISH COLUMBIA CALIFORNIA INSTITUTE OF TECHNOLOGY

UNIVERSITY OF CALIFORNIA MONTANA STATE UNIVERSITY

UNIVERSITY OF NEVADA

NEW MEXICO STATE UNIVERSITY

OREGON STATE UNIVERSITY

UNIVERSITY OF OREGON

OSAKA UNIVERSITY

UNIVERSITY OF SOUTHERN CALIFORNIA
STANFORD UNIVERSITY

UNIVERSITY OF TOKYO

UNIVERSITY OF UTAH

WASHINGTON STATE UNIVERSITY

UNIVERSITY OF WASHINGTON

AMERICAN MATHEMATICAL SOCIETY CHEVRON RESEARCH CORPORATION TRW SYSTEMS

NAVAL WEAPONS CENTER 


\section{Pacific Journal of Mathematics}

\section{Vol. 32, No. $1 \quad$ January, 1970}

Robert Alexander Adams, Compact Sobolev imbeddings for unbounded domains ........................................ 1

Bernhard Amberg, Groups with maximum conditions .................. 9

Tom M. (Mike) Apostol, Möbius functions of order k............... 21

Stefan Bergman, On an initial value problem in the theory of two-dimensional transonic flow patterns ................... 29

Geoffrey David Downs Creede, Concerning semi-stratifiable spaces ...... 47

Edmond Dale Dixon, Matric polynomials which are higher

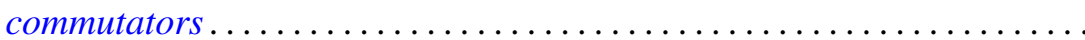

R. L. Duncan, Some continuity properties of the Schnirelmann density.

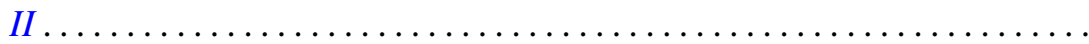

Peter Larkin Duren and Allen Lowell Shields, Coefficient multipliers of $H^{p}$

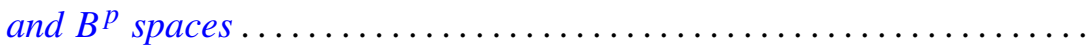

Hector O. Fattorini, On a class of differential equations for vector-valued

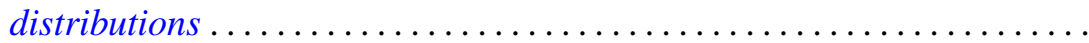

Charles Hallahan, Stability theorems for Lie algebras of derivations. . . . . . 105

Heinz Helfenstein, Local isometries of flat tori ................ 113

Gerald J. Janusz, Some remarks on Clifford's theorem and the Schur

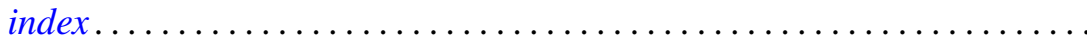

Joe W. Jenkins, Symmetry and nonsymmetry in the group algebras of discrete groups. ...

Herbert Frederick Kreimer, Jr., Outer Galois theory for separable

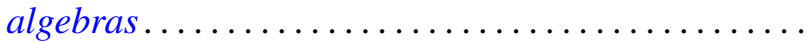

D. G. Larman and P. Mani, On visual hulls

R. Robert Laxton, On groups of linear recurrences. II. Elements of finite order.

Dong Hoon Lee, The adjoint group of Lie groups ...

James B. Lucke, Commutativity in locally compact rings

Charles Harris Scanlon, Rings of functions with certain Lipschitz

$$
\text { properties ............................... }
$$

Binyamin Schwarz, Totally positive differential systems .

James McLean Sloss, The bending of space curves into piecewise helical curves.

James D. Stafney, Analytic interpolation of certain multiplier spaces ...

Patrick Noble Stewart, Semi-simple radical classes.......

Hiroyuki Tachikawa, On left $\mathrm{QF}-3$ rings ...................... 255

Glenn Francis Webb, Product integral representation of time dependent nonlinear evolution equations in Banach spaces.... . . . 